\title{
In Situ Probing of Fast Defect Annealing in Cu and Ni with a High-Intensity Positron Beam
}

\author{
Bernd Oberdorfer, Eva-Maria Steyskal, Wolfgang Sprengel, ${ }^{*}$ and Werner Puff \\ Institute of Materials Physics, Graz University of Technology, A-8010 Graz, Austria \\ Philip Pikart and Christoph Hugenschmidt \\ Physics Department E 21 and FRM II, Technical University Munich, D-85747 Garching, Germany \\ Michael Zehetbauer \\ Physics of Nanostructured Materials, Faculty of Physics, University of Vienna, A-1090 Vienna, Austria \\ Reinhard Pippan \\ Erich Schmid Institute of Materials Science, Austrian Academy of Sciences, and Department Materials Physics, \\ University of Leoben, A-8700 Leoben, Austria \\ Roland Würschum \\ Institute of Materials Physics, Graz, University of Technology, A-8010 Graz, Austria
}

(Received 19 May 2010; published 28 September 2010)

\begin{abstract}
A high-intensity positron beam is used for specific in situ monitoring of thermally activated fast defect annealing in $\mathrm{Cu}$ and $\mathrm{Ni}$ on a time scale of minutes. The atomistic technique of positron-electron annihilation is combined with macroscopic high-precision length-change measurements under the same thermal conditions. The combination of these two methods as demonstrated in this case study allows for a detailed analysis of multistage defect annealing in solids distinguishing vacancies, dislocations, and grain growth.
\end{abstract}

PACS numbers: 81.07.Bc, 61.72.jd, 61.72.Mm, 78.70.Bj

Structural defects such as vacancies, dislocations, and interfaces deliberately introduced into crystalline solids modify their physical properties significantly. Common to these defects is their distinct excess volume as compared to the perfect lattice. Therefore, studies of these defect structures are favorable where experimental methods are involved that directly and specifically detect changes of the atomic volume. Furthermore, in complex defect structures that combine different types of these defects as, e.g., produced by severe plastic deformation [1] the different types can be distinguished according to their characteristic annealing kinetics, i.e., the irreversible volume change as function of temperature and time. For this kind of study two appropriate techniques are available. One technique, dilatometry, is based on macroscopic length-change measurements upon irreversible annealing of defects $[2,3]$. Another technique of atomistic origin is based on the positron-electron annihilation in solids (see, e.g., Refs. [4-7]). In the present Letter we demonstrate a ground breaking improvement of the positron annihilation technique towards application for defect characterization by applying a high-intensity positron beam in combination with the dilatometric technique for in situ studies of fast defect-annealing kinetics.

So far, positron-electron annihilation for defect characterization has been limited to kinetic processes on time scales of hours due to the limitations in positron intensities achievable on a laboratory scale. In the present study this time scale was significantly shortened towards faster defect kinetic studies by the implementation of a high-intensity positron beam at the positron source NEPOMUC of the Heinz-Meier Leibnitz Neutron Source (FRM II Munich, Garching, Germany) [8]. The NEPOMUC facility provides a positron beam with an intensity of $9 \times 10^{8}$ moderated positrons per second at an energy of $1 \mathrm{keV}$. The beam intensity is hence at least 2 orders of magnitude higher than at conventional laboratory beam setups and still higher than comparable large scale facilities $[9,10]$. Using the remoderated beam with $5 \times$ $10^{7}$ positrons/second in situ positron-electron annihilation parameters can now be measured and analyzed on a time scale of a minute, and hence roughly at least 10 times faster than with conventional laboratory based positron annihilation facilities [11]. It allows for specific studies of fast defect-annealing kinetics. In direct combination with high-precision dilatometry it provides a unique and powerful tool for defect analysis as reported in this Letter for the study of ultrafine-grained (UFG) $\mathrm{Cu}$ and $\mathrm{Ni}$ after severe plastic deformation.

Disks of copper (purity 99.995) and nickel (purity $99.99+$ ), $30 \mathrm{~mm}$ in diameter and $10 \mathrm{~mm}$ in height, were severely deformed by high-pressure torsion (HPT) [12] applying a pressure of $2.2 \mathrm{GPa}$. This HPT process yields an ultrafine-grained structure with a mean grain size, $d$, of $270 \mathrm{~nm}$ in the case of $\mathrm{Cu}$ and of $260 \mathrm{~nm}$ in the case of $\mathrm{Ni}$, as determined by scanning electron microscopy (SEM). The 
SEM investigations of the samples also revealed a very uniform microstructure in radial and axial direction; for details see Ref. [13]. The SEM analyses are also supported by microhardness measurements. Only close to the center of the HPT sample a radius dependence of the microstructure is observed; however, this part was entirely avoided in the specimen preparation for the present study. Analyses of the x-ray Bragg profiles [14] and by calorimetry [15] lead to the conclusion that high concentrations of athermally produced lattice vacancies are present at room temperature additional to exceptionally high dislocation densities and large volume fraction of grain boundaries.

The specimens for the dilatometric and positron annihilation experiments were cut from positions where they were subjected to a van Mieses equivalent strain of about $\epsilon=20$ to 30 . For the dilatometric measurements prismshaped specimens were prepared with $3 \times 3 \mathrm{~mm}^{2}$ in cross section and a length $L_{0}$ of $7 \mathrm{~mm}$. For the positron annihilation experiments specimens with dimensions of $8 \times 8 \times$ $1 \mathrm{~mm}^{3}$ were cut. Prior to the positron-beam experiment the metallographically prepared flat specimen surfaces were electropolished in order to remove surface artifacts due to the preparation. The electrochemical etching treatment removed layers of about 100 to $200 \mu \mathrm{m}$ from the $\mathrm{Cu}$ and Ni specimen surface.

The in situ positron-electron annihilation experiments were performed at the coincident Doppler-broadening spectrometer at NEPOMUC, where a beam diameter of less than $1 \mathrm{~mm}$ is achieved at the sample position. In a first temperature calibration run without positron-beam the temperature of the whole specimen stage was determined as a function of time upon a constant energy input as realized by radiation heating. The in situ annealing experiments with the positron beam were then performed on the same setting and the specimen stage was on $-20 \mathrm{kV}$ potential. The energy uptake of the specimen by the remoderated positron beam used was in the order of about $0.1 \times 10^{-6} \mathrm{~W}$ and can be neglected. Therefore, an unambiguous temperature calibration is given. The initial heating starting from room temperature $(298 \mathrm{~K})$ occurred at a rate of about $4 \mathrm{~K} / \mathrm{min}$ which continuously decreased to about $0.5 \mathrm{~K} / \mathrm{min}$ upon reaching the final temperature of $473 \mathrm{~K}$ after $90 \mathrm{~min}$. For the details of the temperature program see the lower and upper abscissas in Fig. 1. During the heating of the specimens the positron-electron annihilation line and its Doppler broadening were continuously monitored with two high-purity Ge detectors (energy resolution $\Delta E=1.35 \mathrm{keV}$ at $511 \mathrm{keV}$ ). Spectra of at least $10^{6}$ counts were obtained in time intervals of $60 \mathrm{sec}$.

The measurements of the relative macroscopic length change $\Delta L / L_{0}$, i.e., the irreversible shrinking of the specimen due to annealing of the volume-associated defects, were performed with a high-precision vertical differentialdilatometer (Linseis L75VD500LT) under Argon (5N) gas flow. The dilatometric measurement of UFG-Ni was
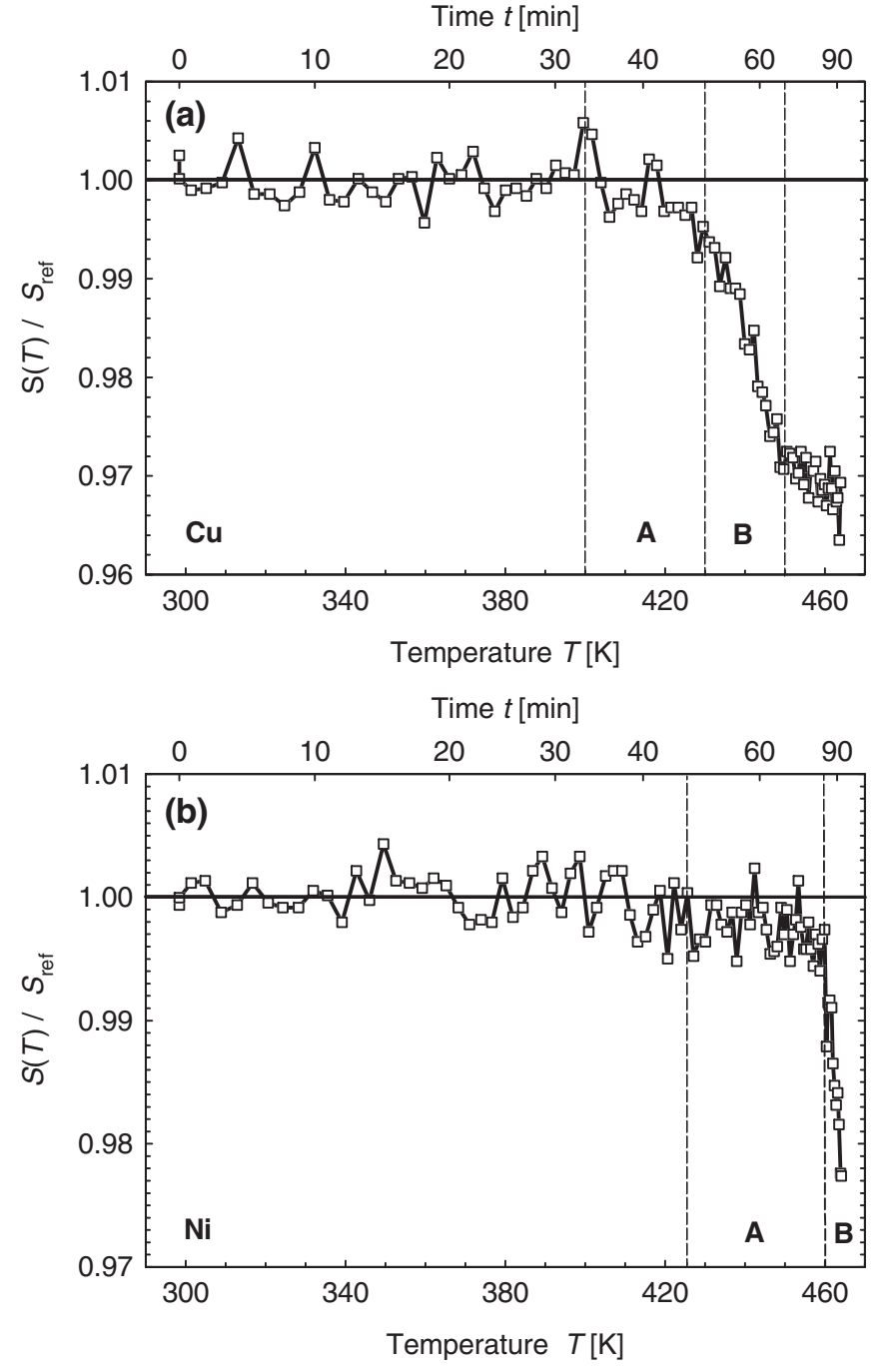

FIG. 1. Temperature dependence of the $S$ parameter upon heating for $\mathrm{Cu}$ [upper panel (a)] and Ni [lower panel (b)]. The data are normalized to the value $S_{\text {ref }}$ of the as-prepared HPTdeformed state. The data points were taken in situ at $60 \mathrm{~s}$ time intervals and each data point is derived from two Doppler spectra with a total of at least $10^{6}$ counts. The upper abscissa shows the corresponding time, $t$.

performed with reference to a well-annealed Ni specimen. The temperature program was identical to the temperature program obtained from the calibration run of the positronbeam experiments. The resulting difference curves yield the relative length change $\Delta L / L_{0}$ caused by the irreversible removal of defects.

The $\mathrm{Cu}$ and $\mathrm{Ni}$ specimens in the HPT-deformed state were also investigated by positron-lifetime experiments at room temperature with a conventional ${ }^{22} \mathrm{Na}$ positron source in sandwich geometry. The results show only one single positron lifetime $\tau=(160 \pm 2)$ ps for $\mathrm{Cu}$ and $\tau=(178 \pm 2) \mathrm{ps}$ for Ni. Therefore, positron saturation trapping can be assumed for the initial specimen state. The values of the positron lifetime have to be considered 
as average values for vacancies and free volumes slightly smaller than a lattice vacancy as typical for dislocations and free volume in grain boundaries (see, e.g., Refs. [16,17]).

In the present study the so-called $S$ parameter was used for the characterization of the annealing behavior. It is most suitable for the study of defect kinetics under the condition of initial prevalent positron saturation trapping. The $S$ parameter represents the integral line parameter of the central part of the Doppler-broadened positron-electron annihilation line which is primarily due to positron annihilation with valence electrons. In a free-volume trapped state the $S$ parameter is enhanced compared to the defectfree state. The electron momentum range from 0 to $6.8 \times$ $10^{-3} m_{0} c$ was used for the calculation of the $S$ parameter where $m_{0}$ denotes the electron rest mass and $c$ the velocity of light. For further details, see, e.g., Ref. [4].

Figure 1 shows the temperature dependence of the $S$ parameter upon heating from room temperature to $483 \mathrm{~K}$ of HPT-deformed $\mathrm{Cu}$ and $\mathrm{Ni}$, respectively. In both cases the $S$ parameter has been normalized to a reference value, $S_{\text {ref }}$, representing the as-prepared, HPT-deformed state. The value of $S_{\text {ref }}$ is the average of the first five data points. The upper abscissa shows the corresponding time, $t$, representing the $T(t)$ heating program which was identical for both measurements. A qualitatively similar behavior is observed for the curves of the normalized $S$ parameter as function of temperature for both $\mathrm{Cu}$ and $\mathrm{Ni}$. At lower temperatures no change is observed for the normalized $S$ parameter upon increasing temperature. Then in a first stage (A) (at $400 \mathrm{~K}$ for $\mathrm{Cu}$ and $425 \mathrm{~K}$ for $\mathrm{Ni}$ ) a slight lowering of the $S$-parameter sets in which is then finally followed by a sharp decrease in a second stage (B) (at $430 \mathrm{~K}$ for $\mathrm{Cu}$ and at $460 \mathrm{~K}$ for $\mathrm{Ni}$ ). In the case of $\mathrm{Cu}$ the value of the normalized $S$ parameter remains constant above $455 \mathrm{~K}$ until the end of the temperature run at $483 \mathrm{~K}$. The corresponding stages are marked in Fig. 1. The first stage (A) is due to annealing of vacancies and dislocations inside the crystallites as discussed in the following in combination with the dilatometric measurements. The second stage (B) results from a temperature driven removal of grain boundaries due to grain growth.

Figure 2 presents the dilatometric measurement, i.e., the irreversible relative length change $\Delta L / L_{0}$ (thick line, right ordinate) as function of temperature for UFG-Ni. The data are superimposed on the temperature dependence of the $S$ parameter. The dilatometric data, as determined with an identical $T(t)$ program, shows a monotonic shrinkage of the specimen starting at around $365 \mathrm{~K}$. Compared to the temperature dependence of the $S$ parameter two distinct stages in similar temperature regions can be distinguished. In a first stage, from 365 to $445 \mathrm{~K}$, a nearly linear shrinkage of the specimen is observed which is then followed in a second stage by a sharp decrease of the length between 445 and $483 \mathrm{~K}$.

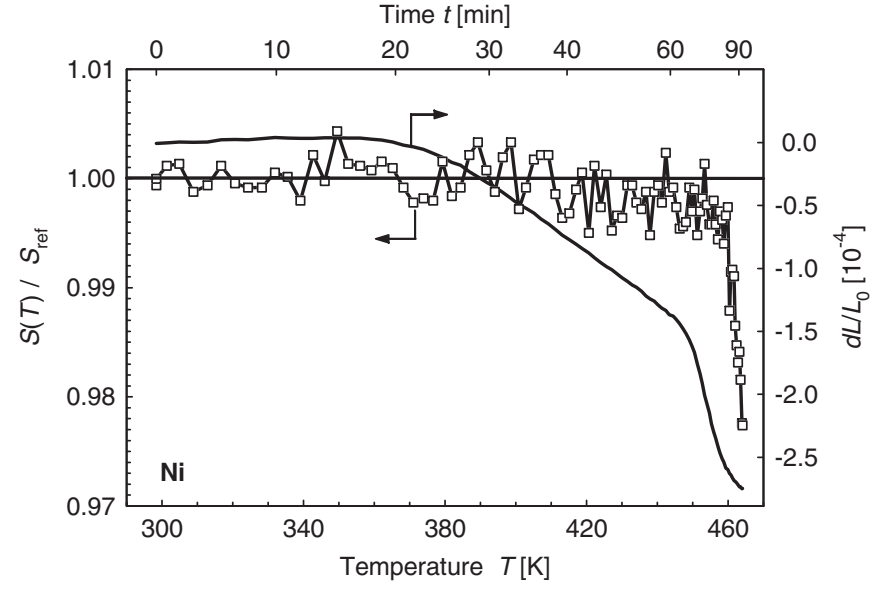

FIG. 2. Comparison of the temperature dependence of the $S$ parameter as determined from positron annihilation [open squares, left ordinate, same as Fig. 1(b)] and superimposed the relative length change $\Delta L / L_{0}$ (thick line, right ordinate) as determined by dilatometry of HPT-deformed Ni. The data points for dilatometry were taken in time intervals of $1 \mathrm{~s}$. In both cases the data were obtained by applying the identical temperature program in two independent measurements on identically prepared specimens. The corresponding time, $t$, valid for both curves is shown on the upper abscissa.

For the discussion of the results we will first focus on the second annealing stage (B). The sharp decrease of the $S$ parameter observed for $\mathrm{Cu}$ at temperatures above $430 \mathrm{~K}$ and for $\mathrm{Ni}$ above $460 \mathrm{~K}$ is caused by a grain growth process as confirmed by scanning electron microscopy. Grain size analyses by SEM of $\mathrm{Cu}$ and $\mathrm{Ni}$ specimens prepared according to the different annealing stages reveal an increase of the mean grain size. From initial grain size of $270 \mathrm{~nm}(\mathrm{Cu})$ and $260 \mathrm{~nm}(\mathrm{Ni})$ the grains grow to sizes of several micrometers in both cases in the temperature range between 430 and $483 \mathrm{~K}$ after applying a similar heating program as for the positron annihilation experiments. As the grain growth is associated with the release of grain boundary volume this results in an immediate effect on the length-change curve as soon as grain growth sets in.

The effect of grain growth resulting in the sharp decrease of the $S$ parameter is noticable at a temperature about $20 \mathrm{~K}$ higher than that for the length change. This is due to the fact that the lowering of the $S$ parameter can only be observed after the grains have grown sufficiently larger than the mean positron diffusion length. It leads to an increased annihilation probability inside the defect-free grains as the positrons are then unable to reach the grain boundaries. Thus, the specimen leaves the state of positron saturation trapping. This conclusion is supported by calculations of diffusion controlled positron trapping at grain boundaries [18]. For grains exceeding a radius of about $200 \mathrm{~nm}$, i.e., for a grain size $d>400 \mathrm{~nm}$, it can be expected that the state of positron saturation trapping is left and positron trapping in the defect-free state starts to dominate. The calculations are based on typical values for 
the positron diffusion coefficient and the specific trapping rate at grain boundaries.

The change of the $S$ parameter in the lower temperature region in the first stage (A) as observed for $\mathrm{Cu}$ at $400 \mathrm{~K}$ and for $\mathrm{Ni}$ at $425 \mathrm{~K}$, however, is slightly more subtle. The nearly linear shrinkage of the Ni specimen in this stage between 365 to $445 \mathrm{~K}$ is caused by annealing of vacancies and isolated dislocations and amounts to a total volume change of $4.5 \times 10^{-4}$ (see Fig. 2). This is confirmed by combined investigations of electrical residual resistivity and transmission electron microscopy [19], and by differential scanning calorimetry $[15,20]$. Furthermore, from electron irradiation experiments on pure $\mathrm{Ni}$ it is well established that $\mathrm{Ni}$ vacancies become mobile at temperatures around $360 \mathrm{~K}[21]$.

In combination with the dilatometric results the occurrence of stage (A) for $\mathrm{Cu}$ and $\mathrm{Ni}$ is attributed to a change of the dominating positron trapping site. In the case of saturation trapping, the measured $S$ parameter represents an average value of the specific $S$ parameters of the different defect types weighted with the respective trapping rates. Therefore, from careful measurements of relative variations of the $S$ parameter, changes in the dominating defect type can be deduced. In the present case, the dominant defect type changes from an initial prevalent saturation trapping at vacancies and free dislocations to a state of dominant trapping at interfaces, i.e., grain boundaries. This conclusion is confirmed by calculations based on a positron trapping model for point defects and grain boundaries in polycrystalline materials [18]. Assuming a specific vacancy trapping rate in metals of $\sigma_{V}=5 \times 10^{14} \mathrm{~s}^{-1}$ and a vacancy concentration as deduced from the dilatometric measurements of $c_{V}=4.5 \times 10^{-4}$ then a vacancy trapping rate of $\sigma_{V} c_{V}=22.5 \times 10^{10} \mathrm{~s}^{-1}$ is calculated. This now has to be compared with the positron trapping rate for grain boundaries which according to the model [18] yields $3 \alpha / r=3 \times 10^{10} \mathrm{~s}^{-1}$ if a specific grain boundary trapping rate $\alpha=10^{3} \mathrm{~m}^{2} \mathrm{~s}^{-1}$ and a mean grain radius of $r=$ $100 \mathrm{~nm}$ is assumed. This difference of roughly a factor of 7 in the two trapping rates confirms the transition from dominant positron trapping at vacancies to positron trapping at grain boundary free volume upon annealing of the lattice vacancies.

In conclusion, a complex atomic defect structure in solids as, e.g., present in $\mathrm{Cu}$ and $\mathrm{Ni}$ after severe plastic deformation, has been successfully analyzed by a combination of the atomistic technique of fast in situ temperature dependent positron-electron annihilation and dilatometry. It was realized with a high-intensity positron beam in a first study of its kind and by macroscopic high-precision lengthchange measurements. The combination of these two in situ methods on short time scales as demonstrated in this case study gives a novel experimental approach for an unambiguous identification of structural defects in solids and for basic studies of their physics comprising also fast defect-annealing kinetics. The application of highintensity monoenergetic positron beams to fast defect kinetics studies even at higher temperatures and also with variable energy opens new experimental fields for the application of positron beams [5].

Supported by the FWF Austrian Science Fund (P21009N20) and by the European Commission under the 6th Framework Programme (RII3-CT-2003-505925).

*w.sprengel@tugraz.at

[1] R. Valiev, Nature Mater. 3, 511 (2004).

[2] H.-E. Schaefer, K. Frenner, and R. Würschum, Phys. Rev. Lett. 82, 948 (1999).

[3] B. Oberdorfer, B. Lorenzoni, K. Unger, W. Sprengel, M. Zehetbauer, R. Pippan, and R. Würschum, Scr. Mater. 63, 452 (2010).

[4] P. Hautojärvi, Positron Annihilation in Solids (Springer, Berlin, 1979).

[5] P. Coleman, Positron Beams and their Applications (World Scientific, Singapore, 2000).

[6] R. Würschum, C. Grupp, and H.-E. Schaefer, Phys. Rev. Lett. 75, 97 (1995).

[7] R. Würschum, E. Shapiro, R. Dittmar, and H.-E. Schaefer, Phys. Rev. B 62, 12021 (2000).

[8] C. Hugenschmidt, B. Löwe, J. Mayer, C. Piochacz, P. Pikart, R. Repper, M. Stadlbauer, and K. Schreckenbach, Nucl. Instrum. Methods Phys. Res., Sect. A 593, 616 (2008).

[9] H. Schut, A. van Veen, J. de Roode, and F. Labohm, Mater. Sci. Forum 445-446, 507 (2004).

[10] A. Hawari, D. Gidley, J. Xu, J. Moxom, A. Hathaway, B. Brown, and R. Vallery, in AIP Conf. Proc. 1099, 862 (2009).

[11] S. Dannefaer, V. Avalos, D. Kerr, R. Poirier, V. Shmarovoz, and S.H. Zhang, Phys. Rev. B 73, 115202 (2006).

[12] A. Vorhauer and R. Pippan, Metall. Mater. Trans. A 39A, 417 (2008).

[13] A. Hohenwarter, A. Bachmaier, B. Gludovatz, S. Scheriau, and R. Pippan, Int. J. Mat. Res. 100, 1653 (2009).

[14] E. Schafler, G. Steiner, E. Korznikova, M. Kerber, and M. Zehetbauer, Mater. Sci. Eng. A 410-411, 169 (2005).

[15] D. Setman, M. Kerber, E. Schafler, and M. Zehetbauer, Metall. Mater. Trans. A 41, 810 (2010).

[16] B. Barbiellini, M. J. Puska, T. Korhonen, A. Harju, T. Torsti, and R. M. Nieminen, Phys. Rev. B 53, 16201 (1996).

[17] J.C. Robles, E. Ogando, and F. Plazaola, J. Phys. Condens. Matter 19, 176222 (2007).

[18] B. Oberdorfer and R. Würschum, Phys. Rev. B 79, 184103 (2009).

[19] I. Novak, Master's thesis, Univ. of Vienna, Austria, 1992.

[20] D. Setman, E. Schafler, E. Korznikova, and M. J. Zehetbauer, Mater. Sci. Eng. A 493, 116 (2008).

[21] W. Wycisk and M. Feller-Kniepmeier, J. Nucl. Mater. 69-70, 616 (1978). 